\title{
Multiple Energy Detectors based Cognitive Radio Networks using Adaptive Double-Threshold Scheme
}

\author{
Jun Hee Lee ${ }^{1}$, Ashish Bagwari ${ }^{2}$ and Geetam Singh Tomar ${ }^{3}$ \\ ${ }^{1}$ Conversing Technology, Hoseo Graduate School of Venture, 70, \\ Gasan Digital 1-ro, Geumcheon-gu, Seoul, Korea \\ ${ }^{2}$ Department of Electronics and Communication Engineering, Uttarakhand Technical \\ University, Dehradun, India \\ ${ }^{3}$ Department of Electrical Engineering, University of West Indies, St. Augustine, \\ Trinidad, and MIR Labs, Gwalior, India \\ 1junhee-lee65@naver.com, ${ }^{2}$ ashishbagwari@ieee.org, ${ }^{3}$ gstomar@ieee.org
}

\begin{abstract}
Cognitive radio is an enabling technology to solve the spectrum scarcity problem in wireless communication. Spectrum sensing is one of the most important functions in Cognitive Radio $(C R)$ implementation \& use to sense the unused spectrum opportunistically. In this paper, we propose Multiple Energy Detectors (MED) utilizing Adaptive Double-Threshold (ADT) for spectrum sensing. In the present framework of CR networks, each CR node is equipped with $M E D$ and each energy detector with a single antenna. Numerical results show that the proposed scheme having the number of antennas $(\mathrm{Nr})=3$ outperforms conventional energy detector by $8.3 \%$ at $-8 d B$ Signal to Noise Ratio (SNR) in terms of probability of detection alarm. When the number of energy detectors $(M)=3$, the signal detects at a very low signal to noise ratio $(S N R)$ i.e. - $9 d B$. Therefore, as the number of energy detectors $(M)$ increases, the detection performance improves and overcomes the sensing failure problem.
\end{abstract}

Keywords: Cognitive radio networks, Multiple energy detectors, Adaptive double-threshold, Spectrum sensing

\section{Introduction}

In cognitive radio systems, the unlicensed users can utilize the licensed frequencies while the Primary User (PU) is absent. For achieving good spectrum sensing performance, many spectrum sensing methods have been studied in CR networks [1], [2], the most widely studied Energy Detection (ED) method [3]. Its implementation is easier than others. But it also brings several challenges, like the presence of a single detector for spectrum sensing in CR which arise the question over CR reliability and performance, and noise uncertainty [4] which increases the difficulty in setting the optimal threshold for a CR and thus degrades its sensing performance due to sensing failure problem [5].

The purpose of using multiple antennas is to mitigate fading \& shadowing effects in the wireless channel. In [6], authors described MED based on Square Law Combining (SLC) receive diversity scheme model using a single threshold for spectrum sensing. This scheme

Article history:

Received (August 10, 2013), Review Result (October 11, 2013), Accepted (November 10, 2013) 
provides redundancy and improves detection performance, but does not overcome the spectrum sensing failure problem.

In this paper, it is shown that the reliability and failure problem of spectrum sensing can be improved by the CR by using multiple energy detectors with an ADT scheme. This paper presents an analytical model called Multiple Energy Detectors (MED) based on a selection combiner (SC) scheme using an adaptive double threshold. The proposed model overcomes the redundancy problem as well as the sensing failure problem with the help of an adaptive doublethreshold scheme.

The motivation of this research paper is to provide a reliable system with improved spectrum sensing performance. Suppose that a CR has single ED, and ED gets fail, in this situation CR cannot work, which is a serious problem in CR networks. Because of the presence of the number of EDs chances of CR failure is minimum. The detection threshold is made adaptive to the fluctuation of the received signal power in each local detector of the CR user. Therefore, this MED is responsible for reducing the chances of CR failure, enhancing reliability, improving performance, processing speed, mitigating sensing failure problems, and can achieve a robust ED in which performance will not be degraded due to noise uncertainty. In this paper, it is shown that the reliability and failure problem of spectrum sensing can be improved by the CR by using multiple energy detectors.

The rest of the paper is organized as follows: Section II presents a system description. Section III describes the proposed system model. Section IV presents the numerical results and analysis. Finally, Section V concludes the paper.

\section{System Description}

CRs utilize unused channels of PU's signal and spectrum sensing mechanism allows them to determine the presence of a PU. In this method, the locations of the primary receivers are not known to the CRs as there is no signaling between the PUs and the CRs. To detect the PU signal, we have used the following hypothesis for the received signal [1]

$$
x(n)= \begin{cases}w(n), & H_{0} \\ s(n) h(n)+w(n), & H_{1}\end{cases}
$$

In the testing, $x(n)$ shows signal received by CR user, $s(n)$ is the PU licensed signal, $w(n) \sim \square$ $\left(0, \sigma_{w}{ }^{2}\right)$ is the Additive White Gaussian Noise (AWGN) with zero mean and variance $\sigma_{w}{ }^{2}, h(n)$ denotes the Rayleigh fading channel gain of the sensing channel between the PU and the CR user. $H_{0}$ is the null hypothesis which indicates that $\mathrm{PU}$ is absent and $H_{l}$ is the alternative hypothesis which indicates that PU is present.

\subsection{Energy Detector}

For the detection of unknown deterministic signals corrupted by the additive white Gaussian noise, an ED is derived in [7], which is called conventional energy detector (CED) [7], [8]. This is an easily implemented detector for the detection of unknown signals in spectrum sensing. It collects the test statistic and compares it to a threshold $(\lambda)$ to decide whether the PU signal is present or absent. The test statistic is given by [9]

$$
X=\frac{1}{N} \sum_{n=1}^{N}|x(n)|^{2},
$$


Where $x(n)$ is the received input signal, $\mathrm{N}$ is the number of samples, $\mathrm{X}$ denotes the energy of the received input signal, which is compared with the threshold to make the final decision. The threshold value is set to meet the target probability of false alarm $\left(\mathrm{P}_{f}\right)$ according to the noise power. The probability of detection $\left(\mathrm{P}_{d}\right)$ can be also identified. The expression for $\mathrm{P}_{f}$ and $\mathrm{P}_{d}$ can be defined as [8].

$$
\begin{gathered}
P_{f}=P_{r}(X<\lambda)=Q\left(\frac{\lambda-N \sigma_{\omega}^{2}}{\sqrt{2 N \sigma_{\omega}^{4}}}\right), \\
P_{d}=P_{r}(X \geq \lambda)=Q\left(\frac{\lambda-N\left(\sigma_{S}^{2}+\sigma_{\omega}^{2}\right)}{\sqrt{2 N\left(\sigma_{S}^{2}+\sigma_{\omega}^{2}\right)^{2}}}\right),
\end{gathered}
$$

Where $\sigma_{\omega}{ }^{2}$ and $\sigma_{S}^{2}$ are the noise variance and signal variance respectively. $Q($ ) denotes Gaussian tail probability Q-function. Now, the total error rate $\left(P_{e}\right)$ is the sum of the probability of false alarm and the probability of missed detection. Thus, the total error rate is given by

$$
P_{e}=P_{f}+\left(1-P_{d}\right)
$$

Where, $\left(1-P_{d}\right)$ shows the probability of missed detection $\left(P_{m}\right)$.

\subsection{Spectrum sensing method with adaptive double-threshold}

In energy detection (ED) based spectrum sensing [10], noise uncertainty increases the difficulty in setting the optimal threshold for a CR and thus degrades its sensing reliability [5], Also this may not be optimum in low SNR conditions where the performance of fixed single threshold $(\lambda)$ based detector can vary from the targeted performance metrics substantially. To mitigate the spectrum sensing failure problem, in [11] authors proposed a two-threshold-based method, but the method requires additional requirements like multiple CRs with FC (fusion center). To eliminate these problems effectively, we propose an adaptive double-threshold scheme having two thresholds $\lambda_{1}$ and $\lambda_{2}$ are used to make the decision.

In [Figure 1], the area comes under the upper bound $\left(\lambda_{1}\right)$ and the lower bound $\left(\lambda_{2}\right)$ is known as a confusing region. In this region detection between noise and PU, the signal is difficult using a single threshold. In the proposed adaptive double-threshold scheme, the upper bound threshold $\left(\lambda_{1}\right)$ is selected according to the maximum noise variance, and the lower bound threshold $\left(\lambda_{2}\right)$ is selected according to the minimum noise variance. Further, the confused region is divided into four equal levels. If detected energy values $(X)$ fall in the confused region it will generate its respective decimal values and are compared with a threshold $(\lambda)$ to make a local decision at a fixed probability of false alarm $\left(P_{f}\right)$ i.e., 0.1 . If values lie outside the confusing region, it will generate 0 or 1 depending upon signal existence. Thus, numerical results show that the proposed scheme enhances the detection performance. 


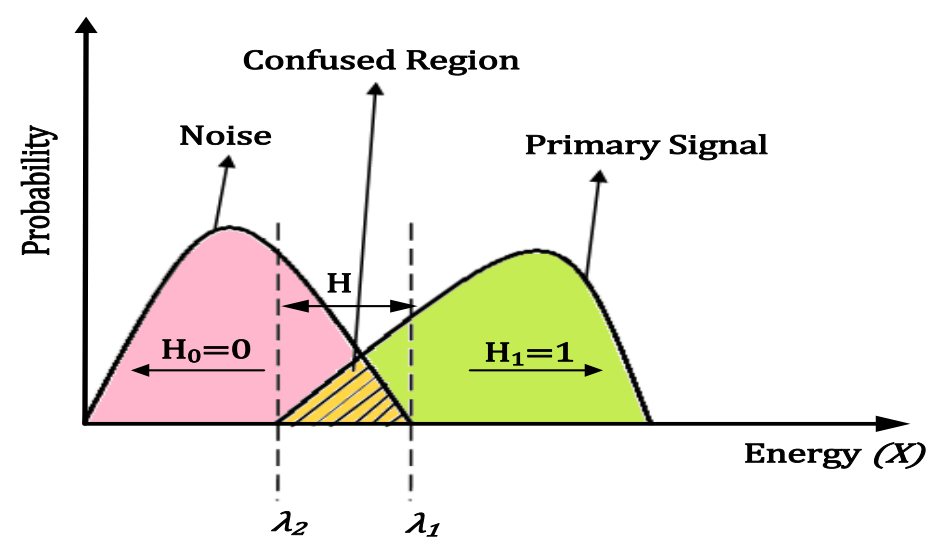

Figure 1. Energy distribution of primary user signal and noise

[Figure 1], shows the energy distribution graph of PU signal and noise. The intersection area is known as the confused region. In this region detection between noise and PU, the signal is difficult using a single threshold. To overcome this problem, we designed the adaptive doublethreshold scheme to determine the local decision at the CR user as following the logic function rule $(L R)$.

$$
L R=\left\{\begin{array}{lr}
H_{0}=0, & X \leq \lambda_{2} \\
H=M, & \lambda_{2}<X<\lambda_{1} \\
H_{1}=1, & \lambda_{1} \leq X
\end{array}\right.
$$

$M$ is the quantization decision, and $X$ denotes received signal energy by the CR user.

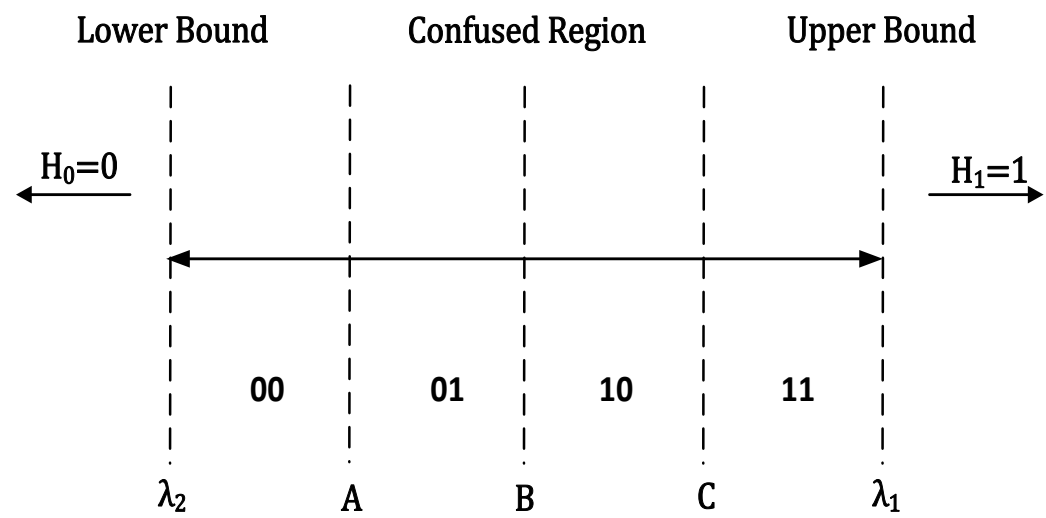

Figure 2. Confused region divided into four equal quantization intervals using two-bit quantization method

A double thresholds method was introduced in [12] to decrease the average number of sensing bits to the common receiver. However, the decreases are achieved at the expense of some sensing performance loss, and it has a sensing failure problem. To eliminate these problems effectively, two thresholds $\lambda_{1}$ and $\lambda_{2}$ are used to help the decision of the cognitive user, as shown in Figure 2. Here, the two-bit quantization method divides the confused region 
into four equal quantization intervals as $\left(\lambda_{2} \mathrm{~A}-\mathrm{AB}-\mathrm{BC}-\mathrm{C} \lambda_{1}\right)$, where $\lambda_{2}, \mathrm{~A}, \mathrm{~B}, \mathrm{C}, \& \lambda_{1}$ are subthresholds $(S T)$ and their values are chosen as

$$
\begin{gathered}
S T=\left\{\begin{array}{l}
A=\lambda_{2}+D \\
B=A+D \\
C=B+D \\
\lambda_{1}=C+D
\end{array}\right. \\
D=\frac{(\text { Uppere bound }- \text { Lower bound })}{\text { No. of Quantization intervals }}=\frac{\left(\lambda_{1}-\lambda_{2}\right)}{4} \\
M= \begin{cases}00, & \lambda_{2}<X \leq A \\
01, & A<X \leq B \\
10, & B<X \leq C \\
11, & C<X<\lambda_{1}\end{cases}
\end{gathered}
$$

In a conventional single threshold case, the false alarm probability $P_{f}$ can be expressed as [8]

$$
P_{f}=Q\left(\frac{\lambda-N \sigma_{\omega}^{2}}{\sqrt{2 N \sigma_{\omega}^{4}}}\right),
$$

Where $Q()$ is the Gaussian tail probability Q-function, and $\sigma_{\omega}{ }^{2}$ is the noise variance. Given the target false alarm probability $\overline{P_{f}}$, the threshold $\lambda$ can be determined as

$$
\lambda=Q^{-1}\left(\overline{P_{f}}\right) \times \sqrt{2 N \sigma_{\omega}^{4}}+N \sigma_{\omega}^{2}
$$

Where $Q^{-1}($ ) denotes the inverse Gaussian tail probability Q-function. Assume that the noise uncertainty in the wireless environment is described as $\left[1 / \rho \sigma_{\omega}{ }^{2}, \rho \sigma_{\omega}{ }^{2}\right]$, where $\rho>1$ is a parameter that quantifies the size of the uncertainty. In the proposed double threshold decision, the upper threshold $\lambda_{l}$ is selected according to the maximum noise variance, and the lower threshold $\lambda_{2}$ is selected according to the minimum noise variance. Therefore

$$
\begin{gathered}
\lambda_{1}=Q^{-1}\left(\overline{P_{f}}\right) \times \sqrt{2 N \rho \sigma_{\omega}^{4}}+N \rho \sigma_{\omega}^{2}, \\
\lambda_{2}=Q^{-1}\left(\overline{P_{f}}\right) \times \sqrt{2 N /\left(\rho \sigma_{\omega}^{4}\right)}+N /\left(\rho \sigma_{\omega}^{2}\right),
\end{gathered}
$$

If detected signals fall inside any one of the quantized intervals, it will generate their respective decimal values $(D V)$ as

$$
D V=\left\{\begin{array}{l}
\text { If } M=00, \text { respective decimal value }-0 \\
\text { If } M=01, \text { respective decimal value }-1 \\
\text { If } M=10, \text { respective decimal value }-2 \\
\text { If } M=11, \text { respective decimal value }-3
\end{array}\right.
$$


Equation (14) shows the decimal values $(D V)$, which are compared with a threshold $(\lambda)$ to make a local decision at a fixed $P_{f}$ i.e. 0.1 . Outside the confused region, it will generate 0 or 1 depending upon signal existence.

\section{Proposed system model}

\subsection{Multiple energy detectors with adaptive double-threshold (MED_ADT)-}

[Figure 3], shows the system model of the proposed multiple ED with adaptive doublethreshold (MED_ADT). Each ED having a single antenna provide a promising solution to improve bit error rate, reducing multipath and shadowing effects of the wireless channel, making the process fast, and improving system reliability. There is one PU that contains a single antenna, $N_{r}$ numbers of energy detectors are implemented at each CR user and each ED has single antennas, hence there is an $N_{r}$ number of antennas as well. $N$ is some samples transmitted by PU. In figure 3, Maximal-ratio combining (MRC) scheme is not considered since it has spectrum sensing overhead due to channel estimation. Moreover, a combining scheme based on the sum of the decision statistics of all antennas in the CR is not analytically tractable. Therefore, we assume that each CR contains a selection combiner (SC) that outputs the maximum value out of $N_{r}$ decision statistics calculated for different diversity branches as $x=\max \left(x_{1}, X_{2}, X_{3}, \ldots, X_{N r}\right)$. The output of the SC is applied to an adaptive double-threshold-based energy detector which decides a spectrum hole.

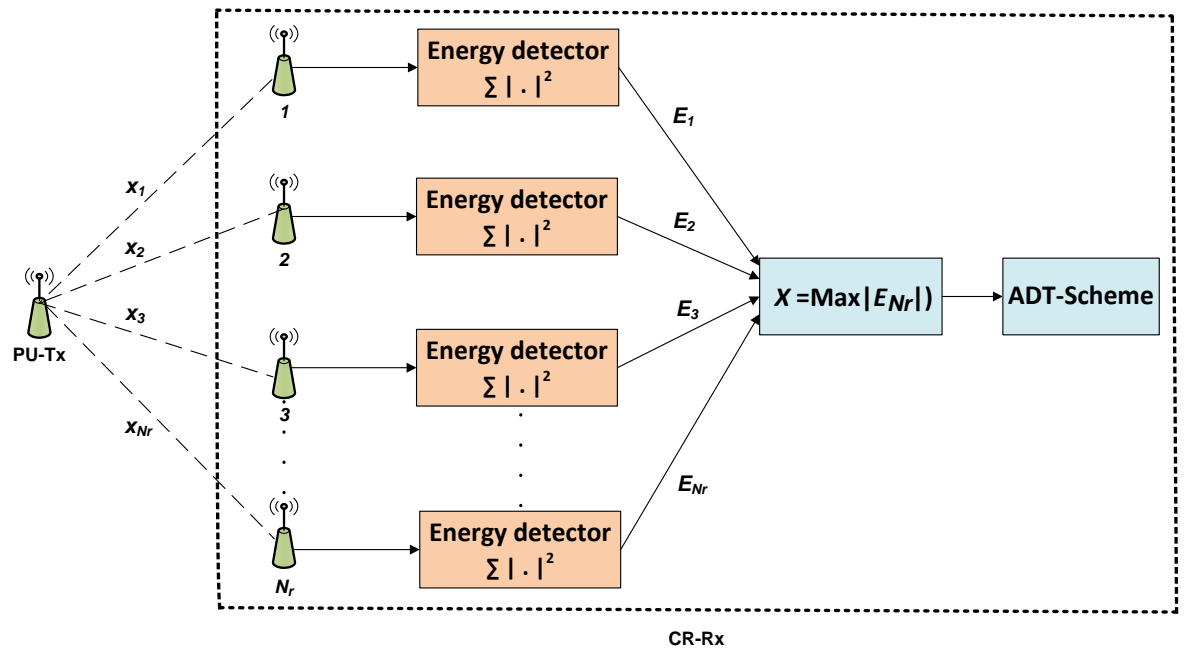

Figure 3. Proposed model: multiple energy detectors with an adaptive double-threshold scheme

Suppose, $x_{j}(k)$ is the received signal at $j^{\text {th }}$ antenna for $k^{\text {th }}$ data stream, the sensing channel between PU and CR is assumed to be Rayleigh fading channel, $N$ is the total number of samples to be sensed by CR and $N_{r}$ is the number of energy detectors. Hence, the overall output of a MED_ADT is as follows:

$$
\operatorname{MED} \_A D T_{\mathrm{o} / \mathrm{p}}=\sum_{j=1}^{N_{r}} E_{j} \text {, }
$$




$$
\begin{gathered}
\text { Where, } E_{j}=\sum_{k=1}^{N}\left|x_{j}(k)\right|^{2}, \\
\text { MED_ADT }_{\mathrm{o} / \mathrm{p}}=\max \sum_{j=1}^{N_{r}}\left[\sum_{k=1}^{N}\left|x_{j}(k)\right|^{2}\right],
\end{gathered}
$$

It is seen from [Figure 3] that individual EDs are allocated to individual antennas. Now we consider the ED branch which has maximum gain and compares it with a threshold using an adaptive double-threshold (ADT) scheme.

[Figure 4], shows the internal architecture of the energy detector with adaptive doublethreshold (ED_ADT). The square-law device detects the signal and shows signal energy $(X)$. After the square-law device, we have two portions named as upper portion and lower portion. In the upper portion, if detected energy values $(X)$ are greater than $\lambda_{l}$, it will show $H_{l}$ (signal presented), or less than $\lambda_{2}$ shows $H_{0}$ (signal absent). But, if detected energy values $(X)$ fall between $\lambda_{1}$ and $\lambda_{2}$ then it will follow the lower portion and use the quantization method to generate its respective decimal values (DV) as shown in equation (14).

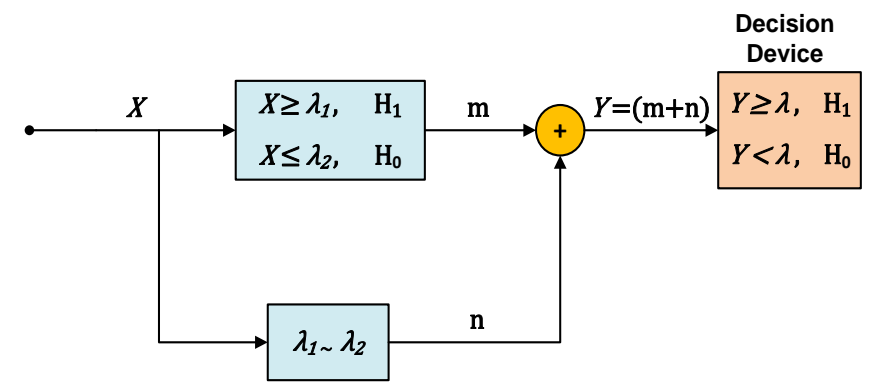

Figure 4. Internal architecture of adaptive double-threshold (ADT) scheme

If detected energy values $(X)$ fall outside/between $\lambda_{1}$ and $\lambda_{2}$, using equation (6), (9) \& (14) it generates value as

$$
\begin{gathered}
m=\left\{\begin{array}{lr}
0, & X \leq \lambda_{2} \\
1, & \lambda_{1} \leq X
\end{array}\right. \\
n=\{D V, \\
\quad \lambda_{2}<X<\lambda_{1}
\end{gathered}
$$

Where $m$ and $n$ are the output values of the upper part and lower part respectively. After that values of $m$ and $n$ are added using adder.

$$
Y=(m+n)
$$

Finally, the local decision $L$ is expressed using equations (18), (19) \& (20), which is the final output of MED_ADT as follows

$$
L= \begin{cases}1, & \lambda \leq Y \\ 0, & Y<\lambda\end{cases}
$$


Equation (21), comparing the resultant value $(Y)$ to the threshold $(\lambda)$, which is maintaining the overall system probability of false alarm $\left(P_{f}\right) 0.1$. If $Y$ is greater than $\lambda$ signal is present otherwise absent.

The novelty of this paper is that there are multiple energy detectors with an adaptive doublethreshold scheme for sensing the PU signal. This scheme optimizes detection performance and overcomes the sensing failure problem.

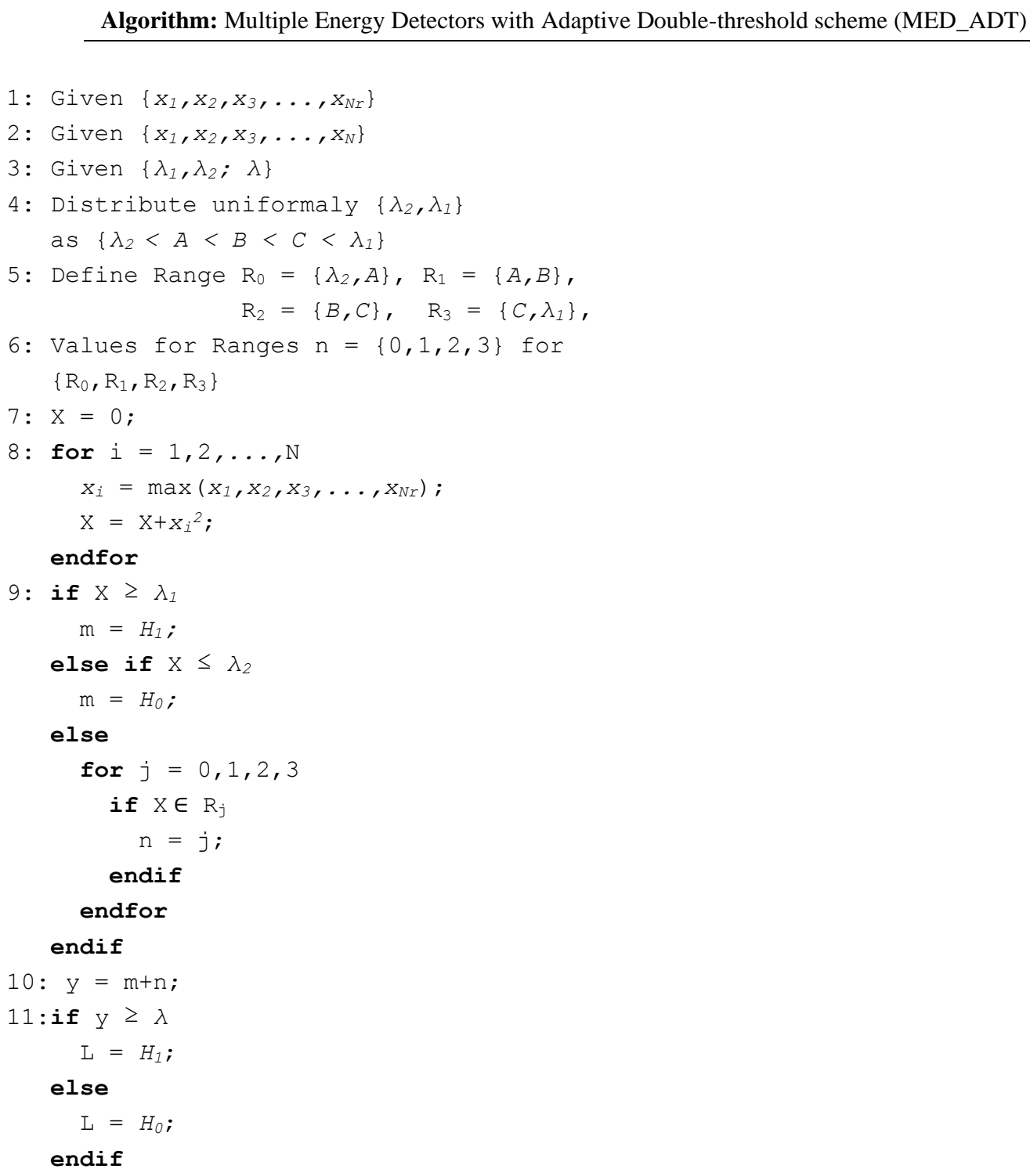




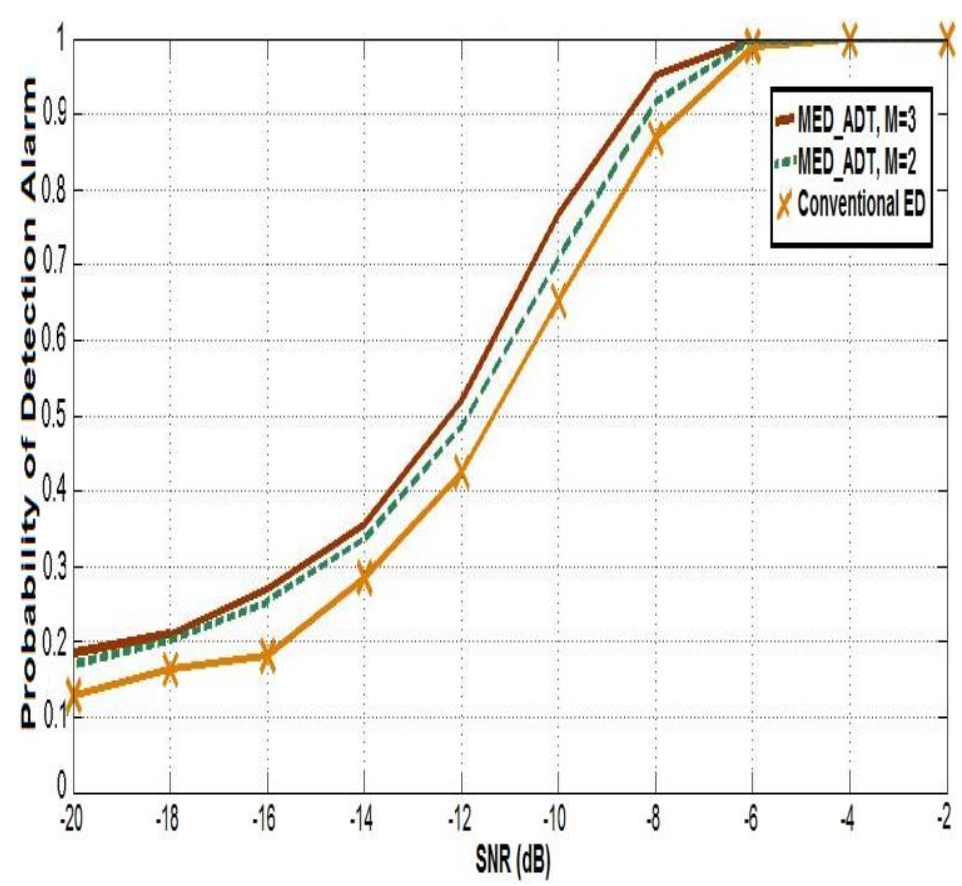

Figure 5. Probability of detection Vs SNR at $P_{f}=0.1$ with $N=1000$, number of energy detectors $N_{r}=$ $1,2,3$, QPSK modulation scheme and rayleigh fading channel

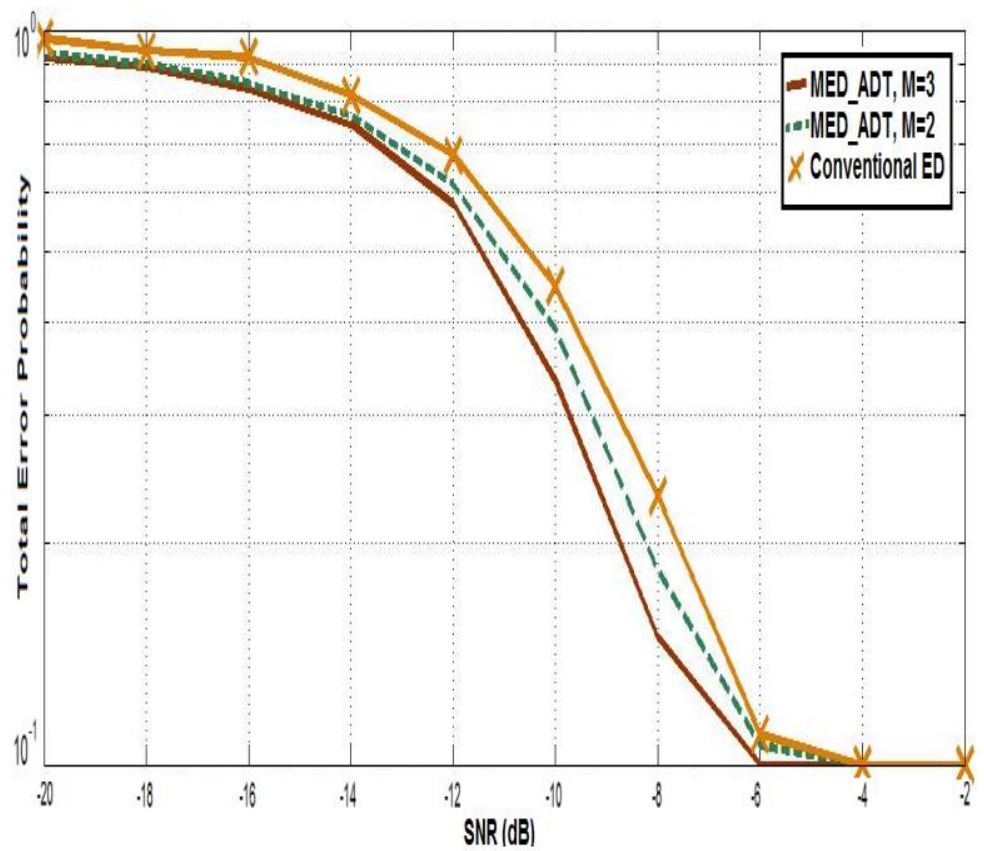

Figure 6. Probability of Error Vs SNR at $P_{f}=0.1$ with $N=1000$, Number of Energy detectors $N_{r}=1$, 2, 3, QPSK modulation scheme and Rayleigh fading channel 


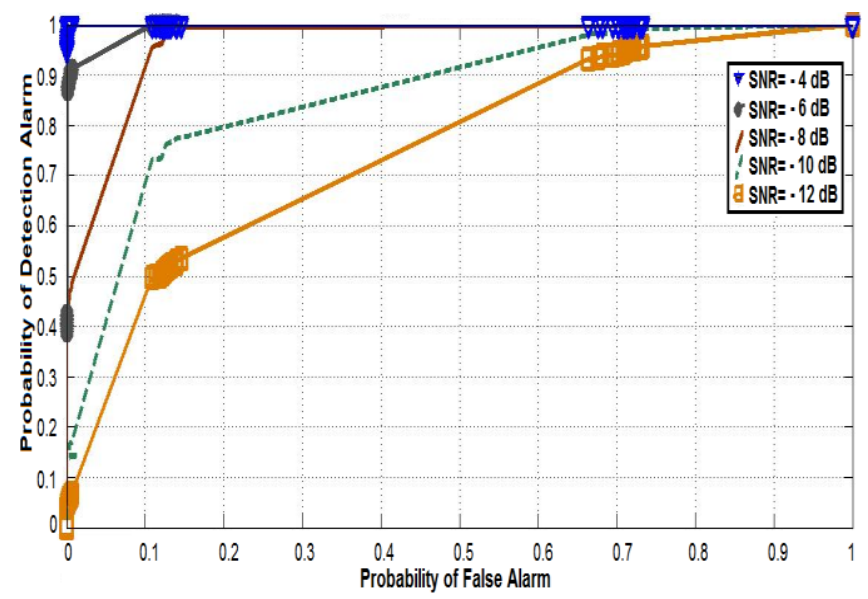

Figure 7. ROC Curves at $\mathrm{N}=1000$, Number of Energy detectors $\mathrm{N}_{\mathrm{r}}=2$, and $\mathrm{SNR}=-4 \mathrm{~dB},-6 \mathrm{~dB},-8$ $\mathrm{dB},-10 \mathrm{~dB},-12 \mathrm{~dB}$

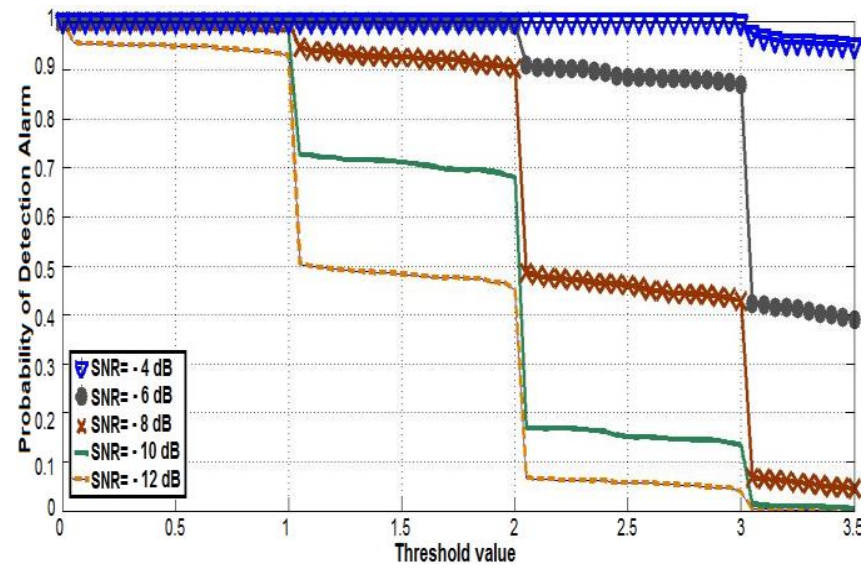

Figure 8. Probability of Detection Vs Threshold values at SNR $=-4 \mathrm{~dB},-6 \mathrm{~dB},-8 \mathrm{~dB},-10 \mathrm{~dB},-12$ $\mathrm{dB}, N_{r}=2$ with $N=1000$, QPSK modulation scheme and Rayleigh fading channel

\section{Numerical results and analysis}

In the presented system model, we have assumed the total number of samples $(N)$ as 1000 , SNR range from $-20 \mathrm{~dB}$ to $-2 \mathrm{~dB}, P_{f}=0.1, N_{r}$ is the number of energy detectors, and QPSK modulation is considered in Rayleigh fading channel.

The probability of detection is critical as it determines how often a primary user is susceptible to potential interference from the cognitive radio system. This is because the time of failures in detecting the presence of a primary user depends on the probability of detection. In cognitive radio networks, the criterion considered so far is in terms of protecting the primary user, i.e., maximizing the probability of detection under the constraint of the probability of false alarm.

It can be seen form figure 5 that when the number of energy detectors increases, probability of detection increases. MED_ADT with $N_{r}=3$ outperforms $N_{r}=1,2$ by $8.3 \% \& 3.5 \%$ at -8 $\mathrm{dB} \mathrm{SNR}$ and $P_{f}=0.1$ respectively. 
[Figure 6] shows that ED_ADT scheme with $N_{r}=3$ has minimum error rate as compared to $N_{r}=1$, 2, i.e. 0.1 at $-6 \mathrm{~dB}$ SNR.

[Figure 7], shows the Receiver Operating Characteristics (ROC) curves. ROC curves exhibit the relationship between sensitivity (probability of detection alarm) and specificity (probability of false alarm) [13] of a spectrum sensing method under different SNR values for MED_ADT when $N_{r}=2$. This implies that when $P_{f}=0.1$ and SNR $=-8 \mathrm{~dB}$, probability of detection is close to 0.9 i.e. 0.9160, which is the spectrum sensing requirement of IEEE 802.22 [14].

It can be seen from figure 8 that there is an inverse relationship between the probability of detection and threshold for a fixed value of SNR. Figure 8 shows as the value of SNR increases, the probability of detection increases up to a level to the threshold. For SNR $=-4 \mathrm{~dB}$, the probability of detection is approximately 1.0 throughout the range of threshold $(\lambda)$, which is better as compared to other SNR values. It implies that when $N_{r}=2$, the proposed MED_ADT can detect PU signal at $-8 \mathrm{~dB}$ SNR for $N=1000$, and $\lambda=2.0$.

\section{Conclusion}

In this paper, we have proposed multiple energy detectors with an adaptive double-threshold (MED_ADT) scheme, which improves system reliability and overcomes the sensing failure problem. Numerical results show that the proposed MED_ADT scheme outperforms conventional energy detectors by $8.3 \%$ at $-8 \mathrm{~dB}$ SNR. This implies that the proposed scheme optimizes detection performance and reduces the total error rate at a very low SNR. Simulation results indicate that the proposed scheme improves detection performance at very low SNR.

\section{Acknowledgments}

The authors wish to thank their parents and God for supporting and motivating for this work because without their blessings this was not possible.

\section{References}

[1] A. Bagwari and B. Singh, "Comparative performance evaluation of Spectrum Sensing Techniques for Cognitive Radio Networks", 2012 Fourth IEEE International Conference on Computational Intelligence and Communication Networks (CICN-2012), (2012) November 3-5; Mathura, Uttar Pradesh, India.

[2] D. Cabric, S. M. Mishra and R. W. Brodersen, "Implementation issues in spectrum sensing for cognitive radios", Proceedings of 2004 Asilomar Conference on Signals, Systems and Computers, vol. 1, (2004) November 7-10; California, USA.

[3] Y. Zeng, Y. C. Liang, A. T. Hoang, and R. Zhang, "A Review on Spectrum Sensing for Cognitive Radio: Challenges and Solutions", EURASIP Journal on Advances in Signal Processing, vol. 2010, (2010), pp. 1-15.

[4] C. Song, Y. D. Alemseged, H. N. Tran, G. Villardi, C. Sun, S. Filin and H. Harada, "Adaptive Two Thresholds based Energy Detection for Cooperative spectrum sensing", Proceedings of 2010 IEEE CCNC, (2012) pp. 1-6.

[5] R. Tandra and A. Sahai, "SNR Walls for Signal Detection", IEEE Journal of Selected Topic in Signal Processing, vol. 2, no.1, (2008), pp. 4-16.

[6] A. Bagwari and G. S. Tomar, "Multiple Energy Detectors based Spectrum Sensing for Cognitive Radio Networks", International Journal of Computer Applications, vol. 62, no. 4, (2013), pp. 11-21.

[7] H. Urkowitz, "Energy detection of unknown deterministic signals", In Proceedings of IEEE, vol. 55, no. 4, (1967), pp. 523-531.

[8] M. López-Benítez and F. Casadevall, "Improved Energy Detection Spectrum Sensing for Cognitive Radio", IET Communications, vol. 6, no. 8, (2012), pp. 785-796.

[9] D. Chen, J. Li, and Jing Ma, "Cooperative Spectrum Sensing under Noise Uncertainty in Cognitive Radio," Wireless Communications, Networking and Mobile Computing, (2008) October 12-14; Dalian, China.

[10] Z. Ling-ling, H. Jian-guo, and T. Cheng-kai, "Novel Energy Detection Scheme in Cognitive Radio", IEEE Conference on Signal Processing, Communications and Computing (ICSPCC), (2011) September 14-16; Xi'an, China.

[11] S. Q. Liu, B. J. Hu and X. Y. Wang, "Hierarchical Cooperative Spectrum Sensing Based on Double Thresholds Energy Detection", IEEE Communications Letters, vol. 16, no. 7, (2012), pp. 1096-1099. 
[12] C. H. Sun, W. Zhang and K. B. Letaief, "Cooperative spectrum sensing for cognitive radios under bandwidth constraints", Proc. 2007 IEEE Wireless Communications and Networking Conference, Vol. 1-9, (2007) March 11-15; Hong Kong, China.

[13] T. Yucek and H. Arslan, "A Survey of Spectrum Sensing Algorithms for Cognitive Radio Applications", IEEE Communication Surveys and Tutorials, vol. 11, no. 1, (2009), pp. 116-130.

[14] T. Do and B. L. Mark, "Improving Spectrum Sensing Performance by Exploiting Multiuser Diversity", Foundation of Cognitive Radio Systems, Prof. Samuel Cheng (Ed.), (2012). 